\title{
Consumo de alcohol, tabaco y cannabis en adolescentes de una población multicultural (Burela, Lugo)
}

\section{Alcohol, tobacco and cannabis consumption in adolescents from a multicultural population (Burela, Lugo)}

\author{
Ainara díaz Geada*, Alicia Busto Miramontes*, **, Francisco Caamaño Isorna*. \\ *Área de Medicina Preventiva y Salud Pública. Universidade de Santiago de Compostela. CIBER-ESP. \\ **Departamento de Medicina Preventiva. Complexo Hospitalario Universitario de Ourense. (CHUO).
}

\section{Resumen}

Las desigualdades en salud en la adolescencia se han asociado a la morbimortalidad de los sujetos. Este estudio pretende evaluar el efecto de género, nacionalidad e inequidades sociales sobre el consumo de alcohol, tabaco y cannabis en adolescentes en un contexto multicultural. Se ha realizado un estudio transversal entre los estudiantes de Educación Secundaria Obligatoria (ESO) de los institutos de Burela (Lugo) (n=238). Se utilizó el cuestionario "Factors de Risc en Estudiants de Secundária" diseñado por la Agència de Salut Pública de Barcelona. Variables independientes: nacionalidad y el dinero disponible semanal. Variables dependientes: expectativas y consumo de alcohol, con consumo de tabaco y marihuana. Se generaron modelos de regresión logística multivariante.

La participación en el estudio alcanzó el 91\%. La proporción de alumnos que ha probado el alcohol aumenta con la edad $(27,3 \%$, $47,7 \%$ y el $75,9 \%)$, como ocurre con el tabaco $(1,8 \%, 7,6 \%$ y $17 \%)$ y el cannabis $(0 \%, 3,1 \%, 7 \%)$. La mayor disponibilidad económica constituye un factor de riesgo para haber probado el alcohol $(\mathrm{OR}=3,01)$, para su consumo de riesgo $(\mathrm{OR}=3,35)$, para haberse emborrachado $(\mathrm{OR}=6,45)$ y para haber probado la marihuana $(\mathrm{OR}=15,30)$. Sexo y nacionalidad no han evidenciado relación con el consumo de ninguna de estas drogas. Los resultados de nuestro estudio muestran que el consumo de alcohol, tabaco y cannabis aumenta con la edad así como con la mayor disponibilidad económica. Los resultados constatan que los alumnos inmigrantes no constituyen un grupo de riesgo y por ello no deben ser estigmatizados.

Palabras clave: Adolescentes; inmigrantes; desigualdades sociales; consumo de sustancias.

\begin{abstract}
Social inequalities have been associated with morbidity and mortality. Gender, ethnic group and inequalities were studied in an adolescent population to analyze alcohol, tobacco and cannabis consumption. We carried out a cross-sectional study of pupils from high schools in Burela (northern Spain) ( $\mathrm{n}=238$ ). We used the "Factors de Risc en Estudiants de Secundária" questionnaire designed by Agència de Salut Pública de Barcelona. Independent variables: nationality and weekly pocket money. Dependent variables: expectations and consumption of alcohol, tobacco and marihuana. Logistic regression was used. Participation in the study reached $91 \%$. The proportion of pupils that have tried alcohol on occasion increases with age $(27.3 \%, 47.7 \%$, $75.9 \%)$, as with tobacco $(1.8 \%, 7.6 \%, 17.0 \%)$, and cannabis $(0 \%, 3.1 \%$, $7.0 \%)$. Higher levels of spending money constitute a risk factor for tasting alcohol $(\mathrm{OR}=3.01)$, for high-risk consumption $(\mathrm{OR}=3.35)$, for getting drunk $(\mathrm{OR}=6.45)$ and for trying marijuana $(\mathrm{OR}=15.30)$. Sex and nationality were not shown to be associated with the use of any of these three drugs. The results of our study show that consumption of alcohol, tobacco and cannabis increases with age and with increased spending money. The data do not support the argument that foreign pupils are a risk group for alcohol consumption, so they should not be stigmatized.

Keywords: Adolescents; immigrants; social inequalities; substance use.
\end{abstract}




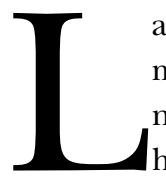

a adolescencia es una etapa de aprendizaje, formación y experimentación, la cual determina buena parte del comportamiento en la vida adulta. Se ha observado que, cuando este período vital se enmarca en un contexto de interacción intercultural, los cambios en los comportamientos de salud pueden acentuarse, convirtiéndolos en una potencial fuente de repercusiones negativas en el bienestar y salud futura de nuestros jóvenes (Bousoño et al., 2017; García-Sánchez et al., 2016; Gonzálvez, Espada, Guillén-Riquelme, Secades, y Orgilés, 2016; Meneses et al., 2009).

En este sentido, la etnicidad o pertenencia a un grupo étnico es un elemento clave de identificación social y cultural (Meneses et al., 2013). También las influencias sociales del grupo de iguales o amigos, junto con los diferentes modelos de rol, la familia y la escuela pueden tener repercusiones en el consumo de sustancias (Giró, 2011; Luengo, Villar, Sobral, Romero y Gómez-Fraguela, 2009; Marsiglia, Kulis, Luengo, Nieri y Villar, 2008; Whal y Eitle, 2010;). Además, se debe señalar la influencia del proceso de aculturación, definido como el intercambio de actitudes culturales y conductas que ocurren cuando personas de distintos entornos socioculturales entran en contacto (Luengo et al., 2009).

Este concepto, aunque tradicionalmente se entendía como la adopción de la cultura dominante, actualmente se tiende a desglosar en diferentes estrategias posibles: la integración o biculturalismo, la asimilación, la separación o retirada y la marginalización o alienación (Luengo et al., 2009). De acuerdo con varias publicaciones, se revela la influencia de este proceso de configuración de la identidad, que podría estar relacionado con el uso de drogas, ya que uno de los factores asociados a estas conductas es que las reproduzca el grupo de iguales (Giró, 2011). Es importante tratar el concepto de manera global y avanzar en el desarrollo de escalas que lo aborden como el multidimensional y complejo proceso que representa (Fosados et al., 2007).

Así pues, resulta fundamental observar más allá de las variables estrictamente personales que intervienen en los adolescentes en el consumo de sustancias. Actualmente, aunque parece haber disminuido el consumo de todas las drogas, el porcentaje de jóvenes españoles de entre 14 y 18 años que han probado alguna vez en la vida el alcohol asciende al $78.9 \%$, el tabaco $38,4 \%$ y la marihuana $29,1 \%$ (Encuesta sobre Uso de Drogas en Estudiantes de Secundaria [ESTUDES], 2016), prevalencias que continúan siendo preocupantes. Así, estos datos convierten el abordaje del consumo de estas sustancias en prioritario para atajar de manera precoz los problemas que en el futuro se puedan derivar de este uso.

Las desigualdades sociales en la etapa inicial del desarrollo son factores contribuyentes a las desigualdades en la vida adulta, hecho que determina la importancia de recoger los determinantes sociales de forma sistemática en los estudios de salud infantil y en la adolescencia (Font-Ribera et al., 2014). La dimensión de la migración no puede entenderse con independencia de la clase social y el género (Borrell y Artazcoz, 2008; Malmusi, Borrell y Benach, 2010) y esta realidad refleja la necesidad de conocer tales comportamientos de riesgo en adolescentes inmigrantes.

Son escasos los estudios realizados en nuestro medio dirigidos a indagar en las desigualdades en salud entre adolescentes que hayan considerado los diferentes grupos étnicos o las diferencias culturales, (Charro Baena, 2015; Font-Ribera et al., 2014; Giró, 2011; Luengo et al., 2009; Marsiglia et al., 2008; Meneses et al., 2009; Meneses et al., 2013;) aun cuando resulta esencial comprender y conocer estas divergencias en aras de incrementar nuestra capacidad de desarrollo de estrategias preventivas en estos subgrupos (Meneses, 2009).

Atendiendo al actual contexto económico, a los cambios en la estructura familiar y social o los flujos migratorios, se hace patente el interés del estudio interrelacionado de desigualdades, emigración y desarrollo adolescente en esta nueva realidad dado que son elementos que repercuten de manera importante en la salud de nuestros jóvenes (Bachman, O’Malley, Johnston, Schulenberg y Wallace, 2011; Blum, Beuhring, Shew, Bearinger y Sieving, 2000; Vega, Aramendi y Garín, 2012). El análisis de patrones de consumo en adolescentes nos permite identificar a los individuos más vulnerables (Caravaca Sánchez, Navarro-Zaragoza, Luna Ruiz-Cabello, Falcón Romero, y Luna Maldonado, 2017; Luengo et al., 2009) de modo que podamos poner en marcha planes preventivos eficaces.

Burela es un municipio del norte de Lugo, Galicia. En los últimos años ha vivido una evolución económica y demográfica verdaderamente rápida y profunda (Oca, 2013). Se trata de un núcleo multicultural donde convive medio centenar de nacionalidades en sus 9.580 habitantes, 8.497 españoles y 1.083 extranjeros (INE 2015). La comunidad mayoritaria es la caboverdiana, a la que más recientemente se han sumado colonias como la peruana, ecuatoriana o senegalesa. Así, esta localidad constituye un vivo ejemplo de convivencia multicultural que pone en la mesa nuevos retos en la búsqueda de una mejor integración social.

El objetivo del presente estudio es determinar el efecto de la nacionalidad y las inequidades sociales sobre el consumo de alcohol, tabaco y cannabis en adolescentes en un contexto multicultural.

\section{Métodos}

\section{Diseño y población del estudio}

Se ha llevado a cabo estudio un transversal o de prevalencia entre estudiantes de $2^{\circ}, 3^{\circ}$ y $4^{\circ}$ de Educación Secundaria Obligatoria (ESO), de 14 a 18 años de los institutos de Burela (Lugo). Toda la población de estudiantes de ESO ha sido reclutada para el estudio $(n=262)$. 


\section{Recogida de datos}

La recogida de datos se realizó utilizando los cuestionarios FRESC (Factors de Risc en Estudiants de SeCundária) (Pérez et al., 2013), diseñado por la Agència de Salut Pública de Barcelona para conocer los comportamientos de riesgo emergentes en estudiantes de ESO. Los principales indicadores que se recogen en el mismo son tales como: variables sociodemográficas, consumo de sustancias adictivas, salud y estado de ánimo, tiempo libre o sexualidad. Se incluyeron dos modelos del cuestionario: uno de $2^{\circ}$ de ESO (13-14) años y otro para $4^{\circ}$ de ESO (15-16) años, que se diferencian en algunas preguntas sobre el consumo de sustancias adictivas, la movilidad o las cuestiones relacionadas con la sexualidad. Para acceder a nuestra población se contactó con los directores y orientadores de los centros educativos, además de contar con la pertinente autorización paterna. Los datos fueron recogidos en las aulas en horario lectivo y en presencia de un docente y un miembro del equipo investigador durante diciembre del año 2015. En todo momento se garantizó la confidencialidad de los datos. Además del cuestionario FRESC, se incluyó el cuestionario KIDMED (Serra-Majem et al., 2004), creado de manera específica para la evaluación de los hábitos dietéticos de niños y adolescentes.

\section{Variables}

\section{Variables independientes}

Nacionalidad. La condición de nacional o inmigrante fue determinada en función de la nacionalidad del padre y de la madre. Fueron considerados inmigrantes aquellos estudiantes cuyos padres nacieron ambos fuera de España.

Dinero disponible semanal. Se preguntó a los estudiantes sobre el dinero disponible semanalmente mediante la pregunta: “¿De cuántos euros has dispuesto a la semana para tus gastos personales?” Las cantidades fueron recodificadas en tres categorías: menos de 10 €; entre 10 y 20 €; y más de $20 €$.

También se consideraron como variables independientes: el sexo y la edad.

\section{Variables dependientes}

Expectativas en relación al consumo de alcohol. Esta variable se midió a través de la pregunta: “¿Piensas que el alcohol hace más divertidas las fiestas? Totalmente de acuerdo/ Bastante de acuerdo/ Bastante en desacuerdo/ Totalmente en desacuerdo". La variable fue recodificada en dos categorías: totalmente o bastante de acuerdo y totalmente o bastante en desacuerdo.

Haber probado el alcohol. Esta variable fue medida mediante la pregunta: “¿Has bebido alguna vez medio vaso de una bebida alcohólica? No, nunca/ Sí, alguna vez/ Sí, en los últimos 12 meses/ Sí, en los últimos 6 meses/ Sí, en los últimos 30 días”. La variable fue recodificada en dos categorías (No vs. Sí).

Consumo intensivo de alcohol. La variable se midió a través de la pregunta: “¿Has tomado alguna vez más de 4 bebidas alcohólicas en una única ocasión? (Considerando ocasión= 4 horas aproximadamente) No, nunca/ Sí, alguna vez/ Sí, en los últimos 12 meses/ Sí, en los últimos 6 meses/ Sí, en el último mes". La variable fue recodificada en dos categorías (No vs. Sí).

Haberse emborrachado. Esta variable fue medida mediante la pregunta: “¿Te has emborrachado alguna vez? No, nunca/ Sí, alguna vez/ Sí, en los últimos 12 meses/ Sí, en los últimos 6 meses/ Sí, en el último mes". La variable fue recodificada en dos categorías. (No vs. Sí).

Haber comprado alguna bebida alcohólica. Variable dicotómica. Se midió a través de la pregunta: “¿Has comprador alguna vez una bebida alcohólica para ti? Sí/ No". La variable fue recodificada en dos categorías (No vs. Sí).

Haber fumado tabaco alguna vez. Variable dicotómica. La cual fue medida mediante la pregunta: “ ¿Has fumado alguna vez? Sí/No".

Haber consumido marihuana. Variable medida a través de la pregunta: “¿Has probado alguna vez la marihuana? No, nunca/ Sí, alguna vez en la vida/Sí, en los últimos 12 meses/Sí, en los últimos 30 días". La variable fue dicotomizada (No vs. Sí).

\section{Análisis estadístico}

Para el análisis descriptivo se han calculado las proporciones y medias. El estudio de las asociaciones se ha llevado a cabo con regresión logística. Se generaron modelos teóricos máximos que incluyeron todas las variables independientes. A partir de ellos se calcularon los modelos finales, en los que se incluyeron todas las variables significativas y aquellas no significativas cuando su exclusión modificara los coeficientes de las restantes variables más de un $10 \%$ (variables confusoras). Los análisis fueron realizados con el paquete estadístico SPSS v22. Por último, el cálculo de proporciones se completó mediante el uso del programa estadístico EPIDAT 3.1 junto con el paquete de análisis estadísticos R.

\section{Resultados}

La participación en el estudio alcanzó el 91\% ( $\mathrm{n}=238)$, de los cuales son foráneos 51 y autóctonos 187 . En cuanto al sexo, la muestra está conformada por un total de 111 chicas y 127 chicos. La Tabla 1 presenta las principales características de los sujetos. En la Tabla 2 se muestran las expectativas y el consumo de alcohol, tabaco y cannabis de los sujetos en función de la nacionalidad y en la Tabla 3 en cuanto al sexo.

En relación a las expectativas sobre el consumo de alcohol, el análisis multivariante indica que ser chico $[\mathrm{OR}=1,83$; IC95\%: 1,04-3,22] y la mayor edad [OR=2.54; IC95\%: 1,23$5,26]$ se asocian a un mayor riesgo de considerar que el alcohol hace más divertidas las fiestas (Tabla 4). La variable edad también se ha mostrado asociada a la proporción de alumnos de 13, 14 y 15 o más años que probaron alcohol alguna vez fue del $27,3 \%, 47,7 \%$ y el $75,9 \%$ respectivamente. 
Cabe señalar que un total de $33.7 \%$ de locales vs. $14.7 \%$ de alumnos inmigrantes han comprado alguna bebida alcohólica $(p=0,035)$ (Tabla 2), de manera que ser local aumenta la posibilidad de comprar alcohol $[\mathrm{OR}=2,95$; IC95\%: 1,04-8,31] (Tabla 4).

La disponibilidad económica ha mostrado asociación con todas las variables estudiadas. Así disponer de más de $10 €$ a la semana incrementa el riesgo de consumir más de 4 bebidas alcohólicas en una única ocasión [OR=3,35; IC95\%:1,34-8,38] así como de emborracharse [OR=6,45; IC95\%:2,31-18,07]. Además, los alumnos con paga semanal superior a $20 €$ presentan la mayor probabilidad de haber probado alcohol [OR=3,01; IC95\%:1,16-7,79] al igual que aquellos de 15 años o más [OR=8,61; IC95\%:3,96-18,71].

La proporción de sujetos que han probado el tabaco alcanzó el 1,8\%, 7,6\% y 17\% en los alumnos 13, 14 y 15 o más años respectivamente, siendo el riesgo para estos últimos 11 veces superior [OR=11,03; IC95\%:1,43-84,73] (Tabla 4).

Por último, y en relación a la marihuana, la proporción de sujetos que han probado esta sustancia asciende del $0 \%$ a los 13 al $7 \%$ a los 15 . Al igual que en las restantes variables dependientes consideradas, la mayor disponibilidad de dinero aumenta significativamente el riesgo de haber probado el cannabis [OR=15,3; IC95\%:1,80-130,43] (Tabla 4). Las variables sexo y la condición de inmigrante no han mostrado relación con el consumo de ninguna de las tres drogas consideradas.
Tabla 1. Descripción de la muestra de estudiantes. Burela, 2015.

\begin{tabular}{ll}
\hline & Total $(\mathrm{n}=238)$ \\
\hline Variables & Porcentaje [IC95\%]+ \\
\hline Nacionalidad & $21,4[16,4-26,6]$ \\
$\quad$ Inmigrante & \\
Sexo & $46,6[40,3-53,3]$ \\
$\quad$ Mujeres & \\
Edad & $23,5[16,8-30,3]$ \\
$\leq 13$ años & $27,7[21,0-34,5]$ \\
14 años & $48,7[42,0-55,5]$ \\
$\geq 15$ años & $14,9[14,8-15,1]$ \\
Media & \\
Disponibilidad económica semanal & \\
$\leq 10$ euros & $55,0[48,7-61,6]$ \\
$10-20$ euros & $23,1[16,8-29,7]$ \\
$\geq 20$ euros & $16,8[10,5-23,4]$ \\
Media & $16,3[13,8-18,9]$ \\
\hline
\end{tabular}

Nota. ${ }^{+} \mathrm{IC}$ : Intervalos de confianza.

Tabla 2. Expectativas y consumo de alcohol, tabaco y cannabis de los sujetos en función de la nacionalidad. Burela, 2015.

\begin{tabular}{|c|c|c|c|c|}
\hline Variables dependientes & $\begin{array}{l}\text { Total } \\
(n=238)\end{array}$ & $\begin{array}{l}\text { Nacionales } \\
(n=187)\end{array}$ & $\begin{array}{l}\text { Inmigrantes } \\
(\mathrm{n}=51)\end{array}$ & p-valor \\
\hline & \multicolumn{4}{|c|}{ Porcentaje / Media } \\
\hline Consideran que el alcohol hace más divertidas las fiestas. & 34,5 & 32,6 & 43,1 & 0,163 \\
\hline Han comprado alguna vez una bebida alcohólica para ellos. & 28,8 & 33,7 & 14,7 & 0,035 \\
\hline Han bebido alguna vez medio vaso de una bebida alcohólica. & 56,3 & 53,5 & 68,6 & 0,054 \\
\hline Han tomado alguna vez más de 4 bebidas alcohólicas en una única ocasión. & 32,0 & 32,3 & 31,4 & 0,929 \\
\hline Se han emborrachado alguna vez. & 24,1 & 21,4 & 31,4 & 0,235 \\
\hline Han fumado tabaco alguna vez. & 10,5 & 9,7 & 14,6 & 0,333 \\
\hline Han probado alguna vez marihuana. & 4,3 & 4,9 & 2,0 & 0,364 \\
\hline
\end{tabular}

Tabla 3. Opiniones y hábitos de acuerdo al género de los estudiantes.

\begin{tabular}{|c|c|c|c|}
\hline & \multicolumn{2}{|c|}{ Porcentaje } & \\
\hline & $\begin{array}{c}\text { Mujeres } \\
n=46,6\end{array}$ & $\begin{array}{c}\text { Hombres } \\
n=53,4\end{array}$ & p-valor \\
\hline Consideran que el alcohol hace más divertidas las fiestas & 39,0 & 61,0 & 0,080 \\
\hline Compraron alguna bebida alcohólica para ellos. & 52,6 & 47,4 & 0,701 \\
\hline Bebieron medio vaso de alguna bebida alcohólica. & 49,3 & 50,7 & 0,080 \\
\hline Bebieron más de cuatro bebidas alcohólicas en una única ocasión. & 28,1 & 35,9 & 0,344 \\
\hline Se emborracharon alguna vez. & 46,9 & 53,1 & 0,795 \\
\hline Fumaron tabaco alguna vez. & 48,0 & 52,0 & 0,933 \\
\hline Probaron cannabis en alguna ocasión. & 3,6 & 4,9 & 0,631 \\
\hline
\end{tabular}


Tabla 4. Influencia de las características de los sujetos sobre las expectativas y prácticas de consumo de alcohol, tabaco y cannabis. Burela, 2015.

\begin{tabular}{|c|c|c|c|c|c|c|c|}
\hline & Odds Ratio (Interval & o de Confianza del & $95 \%)$ & & & & \\
\hline $\begin{array}{l}\text { Variables } \\
\text { explicativas }\end{array}$ & $\begin{array}{l}\text { Considerar que el } \\
\text { alcohol hace las } \\
\text { fiestas divertidas }\end{array}$ & $\begin{array}{l}\text { Haber } \\
\text { comprado } \\
\text { alcohol }\end{array}$ & $\begin{array}{l}\text { Haber bebido } \\
\text { medio vaso }\end{array}$ & $\begin{array}{c}\text { Haberse } \\
\text { emborrachado }\end{array}$ & $\begin{array}{c}\text { Haber tomado más } \\
\text { de } 4 \text { bebidas en } \\
\text { una ocasión }\end{array}$ & $\begin{array}{c}\text { Haber fumado } \\
\text { tabaco }\end{array}$ & $\begin{array}{c}\text { Haber probado } \\
\text { marihuana }\end{array}$ \\
\hline \multicolumn{8}{|l|}{ Sexo } \\
\hline Mujeres & 1 & & & & & & \\
\hline Hombres & $1,83(1,04-3,22)$ & & & & & & \\
\hline \multicolumn{8}{|l|}{ Nacionalidad } \\
\hline Inmigrantes & & 1 & & & & & \\
\hline Nacionales & & $2,95(1,04-8,31)$ & & & & & \\
\hline \multicolumn{8}{|l|}{ Edad } \\
\hline 13 años & 1 & & 1 & & & 1 & \\
\hline 14 años & $1,21(0,53-2,77)$ & & $2,27(1,31-5,01)$ & & & $4,43(0,50-39,08)$ & \\
\hline 15 años & $2,54(1,23-5,26)$ & & $8,61(3,96-18,71)$ & & & $11,03(1,43-84,73)$ & \\
\hline \multicolumn{8}{|l|}{ Paga semanal } \\
\hline$<10$ euros & & & 1 & 1 & 1 & & 1 \\
\hline $10-20$ euros & & & $0,84(0,41-1,70)$ & $6,45(2,31-18,07)$ & $3,35(1,34-8,38)$ & & $15,30(1,80-130,43)$ \\
\hline 20> & & & $3,01(1,16-7,79)$ & $3,07(1,08-8,73)$ & $1,10(0,42-2,86)$ & & $10,42(1,05-103,21)$ \\
\hline
\end{tabular}

Nota. a Ajustado por todas las variables incluidas en la columna.

\section{Discusión}

Los resultados del presente estudio muestran que el consumo de alcohol, tabaco y cannabis aumenta con la edad así como con la mayor disponibilidad económica. Estos datos no muestran asociación entre la condición de inmigrante y el consumo de ninguna de las sustancias consideradas.

Los resultados del estudio se muestran consistentes con los datos obtenidos en la Encuesta sobre Uso de Drogas en Estudiantes de Secundaria (ESTUDES, 2016). Así, nuestros resultados confirman la tendencia de que conforme aumenta la edad, mayor es el riesgo de consumir alguna droga. Sin embargo, nuestros datos no concuerdan con otros estudios que muestran una mayor prevalencia y precocidad de consumo en inmigrantes respecto a autóctonos (Luengo et al., 2009; Meneses et al., 2013). Probablemente esta inconsistencia pueda deberse a dos motivos como: primero, al distinto criterio utilizado a la hora de clasificar a los sujetos como inmigrantes o autóctonos - en nuestro estudio se ha considerado la nacionalidad y no la etnicidad-; y segundo, al hecho de que estos estudios citados analizaron poblaciones de origen mayoritariamente latinoamericano (Luengo et al., 2009; Marsiglia et al., 2008; Meneses et al., 2013; Tortajada et al., 2008) mientras nuestra población inmigrante es mayoritariamente caboverdiana.

La literatura ha apuntado la hipótesis de un posible efecto protector de la etnicidad (Best et al., 2001; Fosados et al., 2007; Marsiglia, Kulis, Hednt y Sills., 2004) aunque este parece cumplirse únicamente en ciertos grupos, como aquellos en los que el peso de la religión, el control paterno (permisividad en los horarios nocturnos, en donde cabe atender a la desigualdad de género), y la abstinencia dentro de la familia pudieran actuar como factores protectores (Giró, 2011).
Debemos puntualizar que, al hablar de etnicidad, la literatura tiende a la autoclasificación de los protagonistas del estudio, aunque se hacen evidentes algunas limitaciones, como en el estudio cualitativo de Charro Baena (2015) en que se observan las dudas que surgen en los alumnos en cuanto a la propia pertenencia étnica. Además se hace necesario atender al proceso de creación de identidades híbridas, construidas desde el contacto en la primera infancia con ambas culturas frente a la asimilación con la consecuente difuminación de las diferencias étnicas (Fosados et al., 2007; Luengo et al., 2009; Marsiglia et al., 2008; Tortajada et al., 2008).

Se sabe que el proceso de aculturación se acentúa en los adolescentes a diferencia de los niños que conviven en la comunidad desde etapas más tempranas del desarrollo. Los inmigrantes de segunda generación tienden a imitar los valores de los autóctonos al haber asimilado sus valores y costumbres (Marsiglia et al., 2004). Algunos autores han señalado el denominado como efecto «White male» (Bachman et al., 2011), refiriéndose al mayor riesgo de éstos en el consumo de sustancias. Entonces, cabe reflexionar sobre el hecho de que nos centremos en la búsqueda de diferencias, corriendo el riesgo de construir nuestras intervenciones sobre variables que no sean susceptibles de ninguna modificación (Blum et al., 2000; Vega et al., 2012).

Con todo, numerosos estudios se inclinan por apoyar la hipótesis de que estas desigualdades no nacen de la nacionalidad o la propia etnia, sino que van a ser dependientes en mayor medida de los recursos económicos de nuestras poblaciones. De manera que la ligazón de la nacionalidad a la desigualdad o la inequidad en salud va a ir de la mano de su nivel socioeconómico (Floyd, Alexandre, Hedden, Lawson y Latimer, 2010; Malmusi et al., 2010). 
En cuanto a las expectativas sobre el consumo de alcohol, nuestros resultados han mostrado que el ser hombre junto con la mayor edad incrementan las expectativas positivas. Estos resultados resultan consistentes con el estudio de Meneses et al., (2013) y se muestran coherentes con los roles tradicionales de género (Borrell y Artazcoz, 2008). No obstante recientes estudios han mostrado similares consumos entre hombres y mujeres (ESTUDES, 2016; Moure-Rodríguez et al., 2016). Este efecto puede ser fruto de cómo la propia sociedad insiste en entender la igualdad y el empoderamiento de la mujer como la absorción de los roles patriarcales. Es decir, es evidente la clara asociación de "ser hombre" con peores hábitos de vida (Borrell y Artazcoz, 2008; Malmusi et al., 2010).

En cuanto a la disponibilidad económica, los datos obtenidos apoyan la hipótesis de que la mayor disponibilidad de recursos va a ser determinante para el mayor consumo. Así, serán los alumnos autóctonos los que, teniendo más recursos, presentarán las más altas prevalencias de consumo de alcohol, tabaco y cannabis. Además, fijándonos en la facilidad de acceso o adquisición de las bebidas alcohólicas, serán nuevamente los autóctonos los que más alcohol compran. Estos resultados se muestran consistentes con los resultados obtenidos en el estudio ESTUDES (2016), así como con el estudio de Moure-Rodríguez et al., (2016) llevado a cabo entre universitarios en nuestro entorno, en el que encontró asociación entre el mayor nivel socioeconómico de la familia y el mayor consumo de alcohol de los estudiantes.

Son cuatro las principales limitaciones de nuestro estudio: 1) El hecho de que el cuestionario utilizado para la recogida de los datos fuese autocumplimentado en la propia aula del instituto, pudo haber provocado que las respuestas de los alumnos se guiaran por lo socialmente aceptable, aun cuando se procuró garantizar la mayor confidencialidad durante el proceso; 2) En la definición de inmigrante no se ha considerado el tiempo de permanencia en el país, lo que pudo haber conducido a una mala clasificación de los sujetos, y así limitar la identificación de la condición de inmigrante como factor de riesgo (Monge et al., 2015); 3) La no inclusión del alumnado de Formación Profesional pudo haber restado validez a los resultados, aunque esta limitación afectará principalmente a los resultados descriptivos y no tanto a los analíticos, ya que no existe base teórica para pensar que las variables independientes consideradas puedan presentar distinto efecto en este grupo poblacional y 4) Probablemente la validez externa de nuestro estudio quede condicionada por la importante variabilidad en la composición y demografía de las distintas comunidades multiculturales en nuestro país. Aun cuando no resulte posible imaginarse un universo de referencia, a nuestro juicio nuestros resultados pueden ayudar a interpretar el consumo de alcohol, tabaco y cannabis en otras poblaciones multiculturales.

En conclusión, los resultados de nuestro estudio muestran que el consumo de alcohol, tabaco y cannabis aumenta con la edad así como con la mayor disponibilidad económica. Estos datos constatan que los alumnos inmigrantes no constituyen un grupo de riesgo para el consumo de estas sustancias y por ello no deben ser estigmatizados. Consideramos, por tanto, que emitir nuevas propuestas para la monitorización de estas desigualdades será fundamental de cara a la mejora de los programas de integración.

\section{Reconocimientos}

A todos los profesionales de los equipos de Atención Primaria que han colaborado. A B. Penabade por su continuo apoyo. A los alumnos y al personal de los Institutos de Educación Secundaria Monte Castelo y O Perdouro de Burela sin cuya ayuda y colaboración el estudio no habría sido posible. Este artículo se ha elaborado sin ninguna financiación.

\section{Conflicto de intereses}

Los autores del artículo declaran que no existe ningún conflicto de intereses.

\section{Referencias}

Bachman, J. G., O’Malley, P. M., Johnston, L. D., Schulenberg, J. E. y Wallace J. M. (2011). Racial/ethnic differences in the relationship between parental education and substance use among U.S. 8th-, 10th-, and 12th-grade students: findings from the Monitoring the Future project. Journal of Studies on Alcohol and Drugs, 72, 279-285.

Best, D., Rawaf, S., Rowley, J., Floyd, K., Manning, V. y Strang J. (2001). Ethnic and gender differences in drinking and smoking among London adolescents. Ethnicity E Health, 6, 51-57. doi:10.1080/13557850123660.

Blum, R. W., Beuhring, T., Shew, M. L., Bearinger, L. H. y Sieving, R. E. (2000). The effects of race/ethnicity, income, and family structure on adolescent risk behaviors. American Journal of Public Health, 90, 1879-1884.

Borrell, C. y Artazcoz, L. (2008). Las desigualdades de género en salud: retos para el futuro. Revista Española de Salud Pública, 82, 241-249.

Bousoño, M., Al-Halabí, S., Burón, P., Garrido, M., Díaz-Mesa, E.M., Galván, G.,... Bobes, J. (2017). Substance use or abuse, internet use, psychopathology and suicidal ideation in adolescents. Adicciones, 29, 97-104. doi:10.20882/ adicciones.811.

Caravaca Sánchez, F., Navarro-Zaragoza, J., Luna Ruiz-Cabello, A., Falcón Romero, M. y Luna Maldonado, A. (2017). Asociación entre victimización por bullying y consumo de sustancias entre la población universitaria en España. Adicciones, 29, 22-32. doi:10.20882/adicciones.827.

Charro Baena, B. (2015). Adolescentes inmigrantes y consumo de alcohol. Miscelánea Comillas. Revista de Ciencias Humanas y Sociales, 72, 140-141. 
Estadística del Padrón Continuo. Varios años (Internet). Madrid: Instituto Nacional de Estadística. Recuperado de http:/ /www.ine.es/nomen2/index.do?accion=busquedaRapida\&subaccion $=\&$ numPag $=0 \&$ ordenAnios $=$ ASC\&nombrePoblacion=Burela\&botonBusquedaRapida=Consultar+selecci\%C3\%B3n

Floyd, L. J., Alexandre, P. K., Hedden, S. L., Lawson, A. L. y Latimer, W. W. (2010). Adolescent drug dealing and race/ethnicity: a population-based study of the differential impact of substance use on involvement in drug trade. The American Journal of Drug and Alcohol Abuse, 36, 87-91. doi:10.3109/00952991003587469.

Font-Ribera, L., García-Continente, X., Davó-Blanes, M $^{\mathrm{a}}$. C., Ariza, C., Díez, E., García Calvente, Ma . M., ...Rajmil L. (2014). El estudio de las desigualdades sociales en la salud infantil y adolescente en España. Gaceta Sanitaria, 28, 316-325. doi:10.1016/j.gaceta.2013.12.009.

Fosados, R., McClain, A., Ritt-Olson, A., Sussman, S., Soto, D., Baezconde-Garbanati, L. y Unger, J. B. (2007). The influence of acculturation on drug and alcohol use in a sample of adolescents. Addictive Behaviors, 32, 2990-3004. doi:10.1016/j.addbeh.2007.06.015.

García-Sánchez, S., Matalí, J.L., Martín-Fernández, M., Pardo, M., Lleras, M., Castellano-Tejedor, C. y Haro, J.M. (2016). Personalidad internalizante y externalizante y efectos subjetivos en una muestra de adolescentes consumidores de cánnabis. Adicciones, 28, 231-241. doi:10.20882/adicciones.887.

Giró, J. (2011). Las amistades y el ocio de los adolescentes, hijos de la inmigración. Papers. Revista de Sociología, 96, 77-91.

Gonzálvez, M.T., Espada, J.P., Guillén-Riquelme, A., Secades, R. y Orgilés, M (2016). Asociación entre rasgos de personalidad y consumo de sustancias en adolescentes españoles. Adicciones, 28, 108-115. doi:10.20882/adicciones.777.

Luengo, M. A., Villar, P., Sobral, J., Romero, E. y Gómez-Fraguela, J. A. (2009). El consumo de drogas en los adolescentes inmigrantes: implicaciones para la prevención. Revista Española de Drogodependencias, 34, 448-479.

Malmusi, D., Borrell, C. y Benach, J. (2010). Migration-related health inequalities: showing the complex interactions between gender, social class and place of origin. Social Science E'Medicine 7, 1610-1619. doi:10.1016/j.socscimed.2010.07.043.

Marsiglia, F. F., Kulis, S., Hecht, M. L. y Sills S. (2004). Ethnicity and ethnic identity as predictors of drug norms and drug use among preadolescents in the US Southwest. Substance Use E Misuse, 39, 1061-1094.

Marsiglia, F. F., Kulis, S., Luengo, M. A., Nieri, T. y Villar, P. (2008). Immigrant advantage? Substance use among Latin American immigrant and native-born youth in Spain. Ethnicity $\mathcal{E}$ Health, 13, 149-170. doi:10.1080/13557850701830356.

Meneses, C., Romo, N., Uroz J., Gil, E., Markez, I., Giménez, S. y Vega, A. (2009). Adolescencia, consumo de drogas y comportamientos de riesgo: diferencias por sexo, etnicidad y áreas geográficas en España. Trastornos Adictivos, 11, 51-63. Meneses, C., Markez, I., Romo, N., Uroz, J., Rua, A. y Laespada, T. (2013). Diferencias de género en el consumo diario de tabaco e intensivo de alcohol en adolescentes latinoamericanos en tres áreas españolas (Andalucía, Madrid y País Vasco). Revista de la Asociación Española de Neuropsiquiatría, 33, 525-535. doi:10.4321/S021157352013000300005.

Monge, S., Ronda, E., Pons-Vigués, M., Vives, C., Malmusi, D. y Gil-González, D. (2015). Limitaciones y recomendaciones metodológicas en las publicaciones sobre salud de la población inmigrante en España. Gaceta Sanitaria, 29, 461-463. doi:10.1016/j.gaceta.2015.07.012.

Moure-Rodríguez, L., Piñeiro, M., Corral Varela, M., Rodríguez Holguín, S., Cadaveira, F. y Caamaño-Isorna, F. (2016). Identifying Predictors and Prevalence of Alcohol Consumption among University Students: Nine Years of Follow-Up. PLoSOne, 11. doi:10.1371/journal. pone.0165514. eCollection 2016.

Oca, L. (2013). Caboverdianas en Burela (1978/2008): migración, relacións de xénero e intervención social. (Tesis doctoral, Universidade de Santiago de Compostela). Recuperado de http://hdl.handle.net/10347/9804.

Plan Nacional sobre Drogas. Encuesta estatal sobre uso de drogas en estudiantes de secundaria (ESTUDES). Madrid: Ministerio de Sanidad y Políticas Sociales; 2016. Recuperado de http://www.pnsd.msssi.gob.es/profesionales/sistemasInformacion/sistemaInformacion/ pdf/2016_ESTUDES_2014-2015.pdf

Pérez, A., García-Continente, X. y Grupo colaborador encuesta FRESC 2012. (2013). Informe FRESC 2012: 25 anysd'enquestes a adolescentsescolaritzats de Barcelona. Barcelona: Agència de Salut Pública de Barcelona.

Serra-Majem, L., Ribas, L., Ngo, J., Ortega, R. M., García, A., Pérez-Rodrigo, C. y Aranceta, J. (2004). Food, youth and the Mediterranean diet in Spain. Development of KIDMED, Mediterranean Diet Quality Index in children and adolescents. Public Health Nutrition, 7, 931-935. doi:10.1079/PHN2004556.

Tortajada, S., Valderrama, J., Castellano, M. 1., Llorens, N., Agulló, V., Herzog, B. y Aleixandre, R. (2008). Consumo de drogas y su percepción por parte de inmigrantes latinoamericanos. Psicothema, 20, 403-407.

Vega, A., Aramendi, P. y Garín, S. (2012). Adolescentes y jóvenes: desde las conductas de riesgo a la inclusión social. Zerbitzuan, 52, 167-178.

Wahl, A. M. y Eitle, T. M. (2010). Gender, acculturation and alcohol use among Latina/o adolescents: a multi-ethnic comparison. Journal Inmigrant Minor Health, 12, 153-165. doi:10.1007/s10903-008-9179-6. 